\title{
Modelling and active damping of powertrain oscillations for RWD electric vehicle
}

\section{Cheng Lin}

Collaborative Innovation Center

of Electric Vehicles in Beijing,

Beijing Institute of Technology,

100081 Beijing, China

Email: lincheng@bit.edu.cn

\section{Shengxiong Sun*}

Collaborative Innovation Center of Electric Vehicles in Beijing, Beijing Institute of Technology, 100081 Beijing, China

and

Faculty of Engineering and IT,

University of Technology,

15 Broadway, Ultimo, NSW 2007 Sydney, Australia

Email: 3120140167@bit.edu.cn

*Corresponding author

\section{Wenfei Jiang}

Shanghai Automobile Gear Works, Huiwang Road 600, Jiading District, 201800 Shanghai, China

Email: jiangwenfei@yeah.net

\begin{abstract}
With the promotion and popularisation of electric vehicles, their driving performance has become an increasingly important issue. As there is no clutch in the powertrain to buffer and absorb the torsional vibrations, vehicle speed oscillations will be caused by various elastic components when the accelerator pedal is stepped or shifted by the driver suddenly. The sudden acceleration input will bring an unpleasant jerk to the vehicle body and accelerates the wear and tear of various parts in the powertrain. In this paper, considering the structure of full electric vehicle, a dynamic model of the powertrain is developed. An optimal feedback controller is proposed based on the state space equation of the vehicle to prevent the driveline from oscillating by actively controlling the motor output torque. The controller parameters are designed based on the optimal control theory. Simulation results demonstrate that the optimal control of motor output torque can reduce the wheel speed oscillations significantly.
\end{abstract}

Keywords: electric vehicle; powertrain; dynamic model; oscillations; optimal control. 
Reference to this paper should be made as follows: Lin, C., Sun, S. and Jiang, W. (2017) 'Modelling and active damping of powertrain oscillations for RWD electric vehicle', Int. J. Powertrains, Vol. 6, No. 4, pp.371-382.

Biographical notes: Cheng Lin received his $\mathrm{PhD}$ in Beijing Institute of Technology, and now he is a professor and his research interests focus on dynamics of electric vehicle, control system of vehicle, lightweight designing etc.

Shengxiong Sun received his MS from Northestern University in 2014, and now he is a doctoral student in Beijing Institute of Technology and University of Technology, Sydney. He currently focuses on the study of control of tortional vibration of powertrain in electric vehicle.

Wenfei Jiang received his BS from Northwestern Polytechnical University in 2014, and received his MS in Beijing Institute of Technology in 2016. He is an engineer of Shanghai Automobile Gear Works up to now.

\section{Introduction}

With the increasingly severe environmental problems and energy crisis, all countries are paying attention to research on alternative energy. Electric vehicle (EV) with its zero emissions, no consumption of gasoline and less noise pollution has won the favour of the whole world. With the promotion and popularisation of electric vehicles, people will surely pay more attention to their driveability, and currently many automotive R\&D vendors also focus on the development of EVs because of their flexibility and low transportation cost, there is no doubt that satisfactory driveability will ensure its multiple utility.

The main parts of an electric vehicle powertrain are motor, transmission, shafts, and wheels, the major difference between the conventional automobile powertrain and electrical vehicle powertrain is that there is no clutch in electric vehicle (as shown in Figure 1). The absence of clutch overcomes the defect of engine, i.e., it cannot stall even it carries great resisting moment of inertia when the EV starts, the cost of manufacturing is reduced, and structure is simplified as well. However, there are no parts to buffer and absorb the torsional vibrations caused by elastic parts when the driver tip in/out suddenly or shift. Early in the beginning of 21st century, scholars of Linköping University in Sweden began the research on gear shifting for the conventional vehicle without a clutch, new driveline-management applications and high-powered engines increase the need to explore the strategies for applying the engine torque in an optimal way (Templin and Egardt, 2011). In order to reduce oscillations, fast and frequent step change of torque input should be avoided, but the passengers will be reluctant to accept the sluggish feeling when appraising the driveability of a car. Therefore, scholars have devoted themselves researching for algorithms which can control the engine output torque actively to damp the torsional vibrations, such as feedforward control (Pham and Bushnell, 2015), model predictive control (Baumann et al., 2006; Caruntu et al., 2016), robust control(Zhang et al., 2014), optimal control (Yu et al., 2015; Berriri et al., 2008) to substitute the conventional Regler Quer Verstellung (RQV) control for diesel engines which is similar to a proportional controller, tracking to the reference speed value given 
by the accelerator pedal input. Furthermore, many research works have been focused especially on the control of electric vehicle, such as fuzzy logic torque control (Song et al., 2015), novel filter using wheel speeds to eliminate oscillations and improve the robustness of EV (Bang et al., 2015), and there are also research works claiming to suppress the oscillation in driveline by controlling the gearshifting (Mousavi et al., 2014). All of the above ideas are based on the same principle which is to control the torque or speed of engine/motor output to damp the driveline oscillations actively, according to the motion state of powertrain, it can calculate the value and direction of torque against trend or direction of speed oscillation as the feedback part timely, so that the power train in closed-loop offers a better performance. However, some of them may hardly be applied in practical situations due to their complexity or defects in internal combustion engine. As the motor installed in electric vehicle has advantages of being controlled easily and responding quickly, it is possible to actively damp the oscillations, especially for the motor-transmission integrated drive system (Fu et al., 2010).

Figure 1 Electric vehicle transmission without a clutch (see online version for colours)
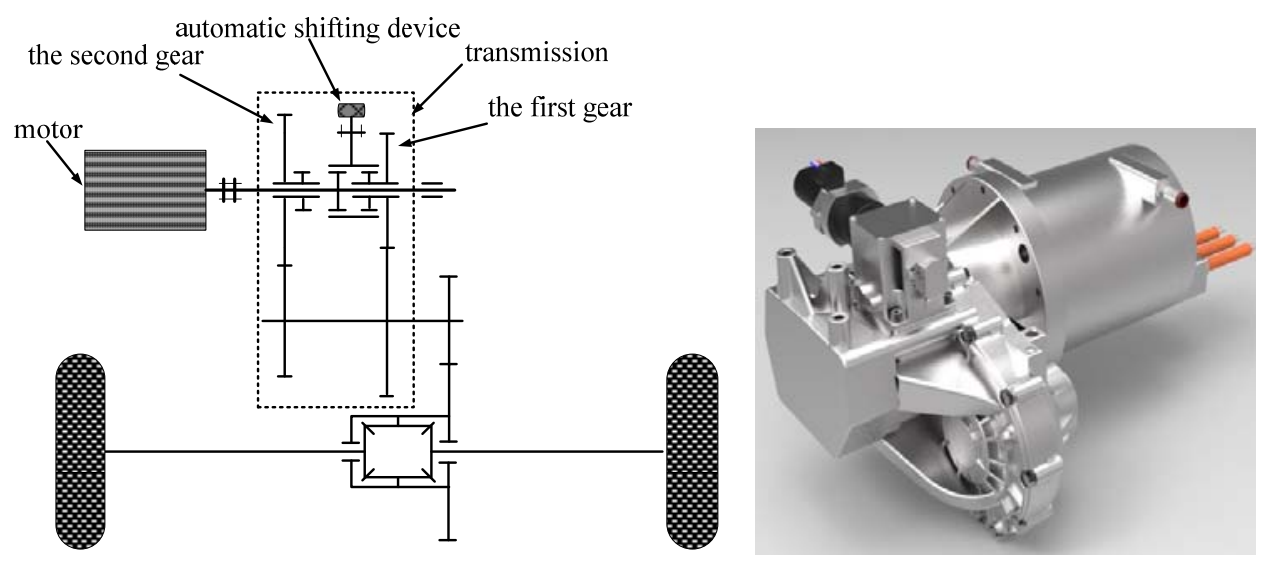

In this paper, based on the structure of electric vehicle, a dynamic model of powertrain without a clutch is established by the Newton's second law, and then state space model of the powertrain is developed. Further, through linearisation of the proposed model and applying the optimal control theory, an extended RQV control algorithm is proposed for the motor control unit (MCU) to damp the oscillations actively. To test the quality of controller, sudden acceleration and deceleration input is provided through the pedal, and simulation results demonstrate that the extended RQV control algorithm can damp the oscillations of EV powertrain significantly.

\section{Driveline modelling}

In this paper a rear-wheel-drive EV is considered, the task of its driveline is to deliver torque from motor to rear wheels. The driveline model consists of a motor rotor, input and output shafts of transmission, final drive, flexible drive shaft, wheels with resistance as shown in Figure 2, instead of the conventional simple drivetrain where the model has two inertias: 
1 engine and transmission

2 wheel and vehicle mass.

Figure 2 Driveline structure (see online version for colours)

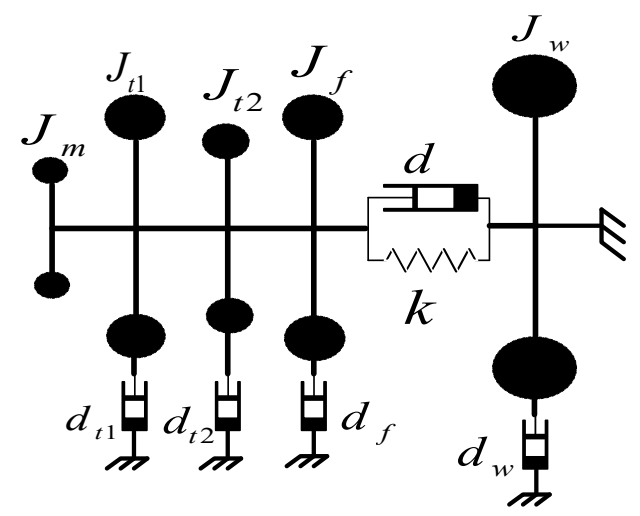

It is assumed that the propeller shaft is stiff due to its short and thick dimensions, the total mass of inertia of the two shafts $J_{t 1}$ and $J_{t 2}$ in transmission is combined to one rotating inertia $J_{t}$, and similarly, $d_{t}$ is the equivalent total damping of respective shafts in transmission. The symbols and subscripts of powertrain mathematical model are shown in Table 1 and Table 2 respectively.

Table 1 Notations

\begin{tabular}{ll}
\hline$J$ & Mass moment of inertia \\
$k$ & Torsional stiffness \\
$d$ & Torsional damping \\
$i$ & Transmission ratio \\
$\theta$ & Torsion angle of drive shaft \\
$T$ & Torque include friction \\
$l$ & Resisting moment \\
$m$ & Gross mass of vehicle \\
$r$ & Wheel radius \\
$\chi$ & Road inclination \\
\hline Table 2 & $\quad$ Subscripts \\
\hline$m$ & Motor \\
$t$ & Transmission \\
$p$ & Propeller shaft \\
$f$ & Final drive \\
$d$ & Drive shaft \\
$w$ & Wheel \\
\hline
\end{tabular}


Based on the structure of the electric vehicle shown in Figure 1 and Figure 2, the equation of motion of all parts is as follows.

\subsection{Motor rotor}

$$
J_{m} \ddot{\theta}_{m}=T_{m}-T_{t}
$$

\subsection{Transmission}

$$
\begin{aligned}
T_{t} i_{t} & =T_{p}, \theta_{m}=\theta_{t} i_{t} \\
J_{t} \ddot{\theta}_{t} & =T_{t} i_{t}-d_{t} \dot{\theta}_{t}-T_{p} \\
J_{t} \ddot{\theta}_{m} & =T_{t} i_{t}^{2}-d_{t} \dot{\theta}_{m}-T_{p} i_{t}
\end{aligned}
$$

\subsection{Propeller shaft}

$$
T_{p}=T_{f}, \theta_{t}=\theta_{p}
$$

\subsection{Final drive}

$$
\begin{aligned}
T_{p} i_{f} & =T_{d}, \theta_{p}=\theta_{f} i_{f} \\
J_{f} \ddot{\theta}_{f} & =T_{f} i_{f}-d_{f} \dot{\theta}_{f}-T_{d}
\end{aligned}
$$

So it can also be described as

$$
J_{f} \ddot{\theta}_{t}=T_{p} \dot{i}_{f}^{2}-d_{f} \dot{\theta}_{t}-T_{d} i_{f}
$$

Combining it with transmission model, equation (3) can be transformed into equation (4), a function of motor speed.

$$
J_{f} \ddot{\theta}_{m}=T_{p} i_{f}^{2} i_{t}-d_{f} \dot{\theta}_{m}-T_{d} i_{t} i_{f}
$$

Combining equation (3) and equation (4), the complete driveline model describing motor, transmission, propeller shaft, and final drive can be obtained as follows:

$$
\left(J_{t} i_{f}^{2}+J_{f}\right) \ddot{\theta}_{m}=T_{t} i_{t}^{2} i_{f}^{2}-d_{t} \dot{\theta}_{m} i_{f}^{2}-d_{f} \dot{\theta}_{m}-T_{d} i_{t} i_{f}
$$

\subsection{Drive shaft}

$$
T_{w}=T_{d}=k\left(\theta_{f}-\theta_{w}\right)+d\left(\dot{\theta}_{f}-\dot{\theta}_{w}\right)=k\left(\frac{\theta_{m}}{i_{t} i_{f}}-\theta_{w}\right)+d\left(\frac{\dot{\theta}_{m}}{i_{t} i_{f}}-\dot{\theta}_{w}\right)
$$

Based on equation (5) and equation (6) above, by replacing $T_{d}$ the equation describing the whole parts of $\mathrm{EV}$ driveline is as follows: 


$$
\begin{aligned}
\left(J_{m}+\frac{J_{t}}{i_{t}^{2}}+\frac{J_{f}}{i_{t}^{2} i_{f}^{2}}\right) \ddot{\theta}_{m} & =T_{m}-\left(\frac{d_{t}}{i_{t}^{2}}+\frac{d_{f}}{i_{t}^{2} i_{f}^{2}}\right) \dot{\theta}_{m}-k\left(\frac{\theta_{m}}{i_{t} i_{f}}-\theta_{w}\right) / i_{t} i_{f} \\
& d\left(\frac{\dot{\theta}_{m}}{i_{t} i_{f}}-\dot{\theta}_{w}\right) / \\
- &
\end{aligned}
$$

\subsection{Wheel}

Vehicle longitudinal force consists of rolling resistance, aerodynamic drag, acceleration resistance and grade resistance.

$$
\begin{aligned}
F_{l} & =F_{r}+F_{w}+F_{j}+F_{i} \\
J_{w} \ddot{\theta}_{w} & =T_{w}-F_{l} r \\
\left(J_{w}+m r^{2}\right) \ddot{\theta}_{w} & =k\left(\frac{\theta_{m}}{i_{t} i_{f}}-\theta_{w}\right)+d\left(\frac{\dot{\theta}_{m}}{i_{t} i_{f}}-\dot{\theta}_{w}\right)-\left(d_{w}+m g c_{r 2} r^{2}\right) \dot{\theta}_{w} \\
& -\frac{1}{2} c_{a i r} A_{L} \rho_{a} r^{3} \dot{\theta}_{w}^{2}-r^{2} m g \frac{\mathrm{d} \theta_{w}}{\mathrm{~d} t}-r m g\left(c_{r 1}+\sin \chi\right)
\end{aligned}
$$

$c_{r 1}, c_{r 2}$ are the coefficient in fitting formula of rolling resistance $F_{r}=m g\left(c_{r 1}+c_{r 2} r \dot{\theta}_{w}\right)$, their values are 0.01 and 0.002 respectively according to (Mitschke and Wallentowitz, 2009), $c_{\text {air }}$ denotes the aerodynamic drag coefficient, $A_{L}$ denotes the vehicle frontal area, $\rho_{\alpha}$ denotes the air density.

The torsion of driveshaft, wheel speed and motor speed are introduced as state variables.

$$
x_{1}=\frac{\theta_{m}}{i_{t} i_{f}}-\theta_{w},{ }_{2}=\dot{\theta}_{w}, x_{3}=\dot{\theta}_{m}
$$

The driveline model equation (7) and equation (9) can be written in the form of state space model seen in equation (10). Here, $u$ is the motor output torque $T_{m}, l$ is the load disturbance.

$$
\dot{\mathbf{x}}=\mathbf{A} \mathbf{x}+\mathbf{B} u+\mathbf{H} l
$$

where $\mathbf{A}, \mathbf{B}, \mathbf{H}, l$ are defined as 


$$
\begin{aligned}
& \mathbf{A}=\left(\begin{array}{ccc}
0 & -1 & \frac{1}{i} \\
\frac{k}{J_{2}} & -\frac{d+d_{2}}{J_{2}} & \frac{d}{i J_{2}} \\
-\frac{k}{i J_{1}} & \frac{d}{i J_{1}} & -\frac{d_{1}+\frac{d}{i^{2}}}{J_{1}}
\end{array}\right) \mathbf{B}=\left(\begin{array}{c}
0 \\
0 \\
\frac{1}{J_{1}}
\end{array}\right) \mathbf{H}=\left(\begin{array}{c}
0 \\
-\frac{1}{J_{2}} \\
0
\end{array}\right) \\
& l=r\left(f m g+\frac{1}{2} c_{a i r} A_{L} \rho_{a} r^{3} \dot{\theta}_{w}^{2}+m g r \frac{\mathrm{d} \theta_{w}}{\mathrm{~d} t}\right) .
\end{aligned}
$$

with

$$
\begin{aligned}
i & =i_{t} i_{f}, J_{1}=J_{m}+J_{t} / i_{t}^{2}+J_{f} / i_{t}^{2} i_{f}^{2}, J_{2}=J_{w}+m r^{2}, d_{1}=d_{t} / i_{t}^{2}+d_{f} / i_{t}^{2} i_{f}^{2}, \\
d_{2} & =d_{w}+m g c_{r 2} r^{2}
\end{aligned}
$$

\section{Controller design}

The active control proposed for controlling the oscillations or jerk in powertrain is based on the conventional RQV speed modulation. The controller is designed to compute a corrective torque that compensates for the oscillations when accelerating and when shifting, torque in transmission should be controlled into a situation suitable for shifting. For damping the speed oscillations of electric driveline, wheel speed is chosen as the measured output of control system:

$$
y=\dot{\theta}_{w}=\mathbf{C x}+e
$$

with $\mathbf{C}=\left(\begin{array}{lll}0 & 1 & 0\end{array}\right), e$ is measurement disturbances. While wheel speed $\dot{\theta}_{w}$ is also the performance output, which is specified by matrix $\mathbf{M}$. The performance output $z$ is defined as

$$
z=\dot{\theta}_{w}=\mathbf{M x}+\mathbf{D u}
$$

where $\mathbf{M}=\left(\begin{array}{lll}0 & 1 & 0\end{array}\right), \mathbf{D}=\mathbf{0}$ if $z=\dot{\theta}_{w}$. In the extended RQV controller, the optimal feedback to minimise the oscillations for EV powertrain is calculated using the states of the system.

The control objective is to minimise the deviations between the wheel speed and desirable reference value $r_{r e f}$, and considering the motor cannot output infinitely small torque $\left(\dot{\theta}_{w}=r_{r e f}\right)$ in engineering practice, the cost function should be extended as follows: 


$$
\lim _{T \rightarrow \infty} \int_{0}^{T}\left(z-r_{r e f}\right)^{2}+\eta\left(u-u_{0}\left(r_{r e f}, l\right)\right)^{2} \mathrm{~d} t
$$

where $r_{\text {ref }}$ is the driver's command as reference speed, and $\eta$ is used as a trade-off between the amplitude of control signal and rise time. When the controller inputs a static value $u_{0}$, the state of system will locate at a stable and static point $\mathrm{z}=r_{r e f} . u_{0}$ is a function of reference speed and load, and when a certain wheel speed is given, the stationary point of driveline is as follows:

$$
\begin{aligned}
& u_{0}\left(\dot{\theta}_{w}, l\right)=\left(\begin{array}{cc}
\left(d_{1} i^{2}+d_{2}\right) & 1 / i
\end{array}\right)\left(\begin{array}{c}
\dot{\theta}_{w} \\
l
\end{array}\right) \triangleq \lambda_{x} \dot{\theta}_{w}+\lambda_{l} l \\
& x_{0}\left(\dot{\theta}_{w}, l\right)=\left(\begin{array}{cc}
d_{2} / k & 1 / k \\
1 & 0 \\
i & 0
\end{array}\right)\left(\begin{array}{c}
\dot{\theta}_{w} \\
l
\end{array}\right) \triangleq \delta_{x} \dot{\theta}_{w}+\delta_{l} l
\end{aligned}
$$

Extended RQV control algorithm is applied to minimise the function (13). However the equation (10) is affine system due to the presence of constant term $l$. To calculate an optimal feedback controller based on LQR algorithm, the model (10) must be linearised around the stationary point, which can be given by equation (14) (Pettersson, 1997)

$$
\Delta \dot{\mathbf{x}}=\mathbf{A} \Delta \mathbf{x}+\mathbf{B} \Delta u
$$

with

$$
\Delta \mathbf{x}=\mathbf{x}-\mathbf{x}_{0}, \Delta u=u-u_{0}, \mathbf{x}_{0}=\mathbf{x}_{0}\left(x_{20}, l\right), u_{0}=u_{0}\left(x_{20}, l\right)
$$

and $x_{20}$ is the initial value of $x_{2}$, the second element in the vector $\mathbf{x}$, and combined with equation (12) the cost function (13) can be transformed into

$$
\lim _{T \rightarrow \infty} \int_{0}^{T}\left(\mathbf{M} \Delta \mathbf{x}+r_{1}\right)^{2}+\eta\left(\Delta u-r_{2}\right)^{2} \mathrm{~d} t
$$

With $r_{1}=\mathbf{M} \mathbf{x}_{0}-r_{r e f}, r_{2}=u_{0}-u_{0}\left(r_{r e f}, l\right)$, and combined with $(\mathbf{A}, \mathbf{B})$, an augmented model of powertrain can be obtained as equation (17). $\sigma$ is a coefficient to control the rate of change for variable $r$, in order to make it stable and controllable, $\sigma$ must be small such that $r_{1,2}$ are slow-varying values described as equation (18).

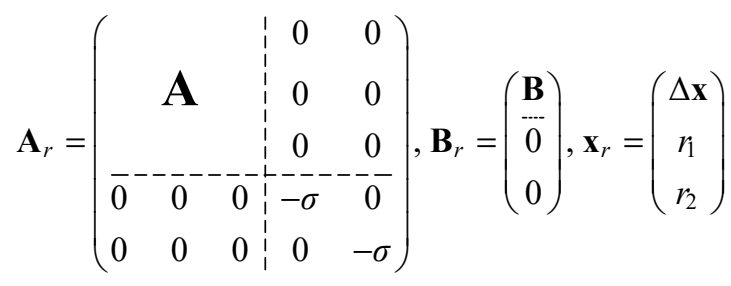

$$
\begin{aligned}
& \dot{r}_{1}=-\sigma r_{1}, \dot{r}_{2}=-\sigma r_{2}
\end{aligned}
$$

Further, the cost function (16) can be transformed into

$$
\lim _{T \rightarrow \infty} \int_{0}^{T}\left(\mathbf{x}_{r}^{\mathrm{T}} \mathbf{Q} \mathbf{x}_{r}+R \Delta u^{2}+2 \mathbf{x}_{r}^{\mathrm{T}} \mathbf{N} \Delta u\right) \mathrm{d} t
$$


with $\mathbf{Q}=\left(\begin{array}{lllll}\mathbf{M} & 1 & 0\end{array}\right)^{\mathrm{T}}\left(\begin{array}{llll}\mathbf{M} & 1 & 0\end{array}\right)+\eta\left(\begin{array}{llllll}0 & 0 & 0 & 0 & 1\end{array}\right)^{\mathrm{T}}\left(\begin{array}{lllll}0 & 0 & 0 & 0 & 1\end{array}\right) \mathbf{N}=\eta\left(\begin{array}{lllll}0 & 0 & 0 & 0 & 1\end{array}\right)^{\mathrm{T}}, R=\eta$.

According to LQR algorithm, when optimal feedback $\Delta u=-\mathbf{K}_{c} \mathbf{x}_{r}$ is used, the cost function (16) is minimum. With $\mathbf{K}_{c}=\mathbf{Q}^{-1}\left(\mathbf{B}_{r}^{\mathrm{T}} \mathbf{P}_{c}+\mathbf{N}^{\mathrm{T}}\right)$, and $\mathbf{P}_{c}$ is the solution of the Riccati equation [equation (20)].

$$
\mathbf{A}_{r}^{\mathrm{T}} \mathbf{P}_{c}+\mathbf{P}_{c} \mathbf{A}_{r}+R-\left(\mathbf{P}_{c} \mathbf{B}_{r}+\mathbf{N}\right) \mathbf{Q}^{-1}\left(\mathbf{P}_{c} \mathbf{B}_{r}+\mathbf{N}\right)^{\mathrm{T}}=0
$$

The control signal $\Delta u$ can also be defined as

$$
\Delta u=-\mathbf{K}_{c} \mathbf{x}_{r}=-\left(\begin{array}{lll}
\mathbf{K}_{c 1} & \mathbf{K}_{c 2} & \mathbf{K}_{c 3}
\end{array}\right) \Delta \mathbf{x}-\mathbf{K}_{c 4} r_{1}-\mathbf{K}_{c 5} r_{2}
$$

and combined with equation (15), the motor output torque $u$ is given by equation (22) to minimise the cost function (16).

$$
u=\mathbf{K}_{0} \mathbf{x}_{20}+\mathbf{K}_{l} l+\mathbf{K}_{r} r_{r e f}-\left(\begin{array}{lll}
\mathbf{K}_{c 1} & \mathbf{K}_{c 2} & \mathbf{K}_{c 3}
\end{array}\right) \mathbf{x}
$$

with

$$
\begin{aligned}
& \mathbf{K}_{0}=\left(\begin{array}{lll}
\mathbf{K}_{c 1} & \mathbf{K}_{c 2} & \mathbf{K}_{c 3}
\end{array}\right) \delta_{x}-\mathbf{K}_{c 4} \mathbf{M} \delta_{x}+\lambda_{x}-\mathbf{K}_{c 5} \lambda_{x} \\
& \mathbf{K}_{r}=\mathbf{K}_{c 4}+\mathbf{K}_{c 5} \lambda_{x} \\
& \mathbf{K}_{l}=\left(\begin{array}{lll}
\mathbf{K}_{c 1} & \mathbf{K}_{c 2} & \mathbf{K}_{c 3}
\end{array}\right) \delta_{l}-\mathbf{K}_{c 4} \mathbf{M} \delta_{l}+\lambda_{l}
\end{aligned}
$$

\section{Simulation results}

The proposed active damping controller is tested, and the obtained results are compared with that of the conventional RQV. The critical parameters of the electric vehicle considered for simulation are presented in Table 3. The test is conducted in the condition when the electric vehicle accelerates rapidly. The comparisons include speed oscillation in powertrain and torque in engaged gears of transmission.

Table 3 Critical parameters

\begin{tabular}{ll}
\hline$m$ & $1,565 \mathrm{~kg}$ \\
$i_{g 1}$ & 2.4375 \\
$i_{f}$ & 4.3125 \\
$d$ & 12.67 \\
$r$ & $0.309 \mathrm{~m}$ \\
$i_{g 2}$ & 1.4815 \\
$k$ & $3,783.5 \mathrm{Nm} / \mathrm{rad}$ \\
$l$ & $276 \mathrm{Nm}$ \\
\hline
\end{tabular}

After the motor starts, the driver needs to step on the accelerator pedal to accelerate the electric vehicle to speed up for shifting, because the motor output always responds rapidly, speed oscillation may occur in the powertrain. In this test, the initial speed of EV is set to $5 \mathrm{Km} / \mathrm{h}$, aerodynamic drag is ignored at low speeds, and no slip occurred when accelerating. 
Figure 3 Drive-shaft speed oscillation

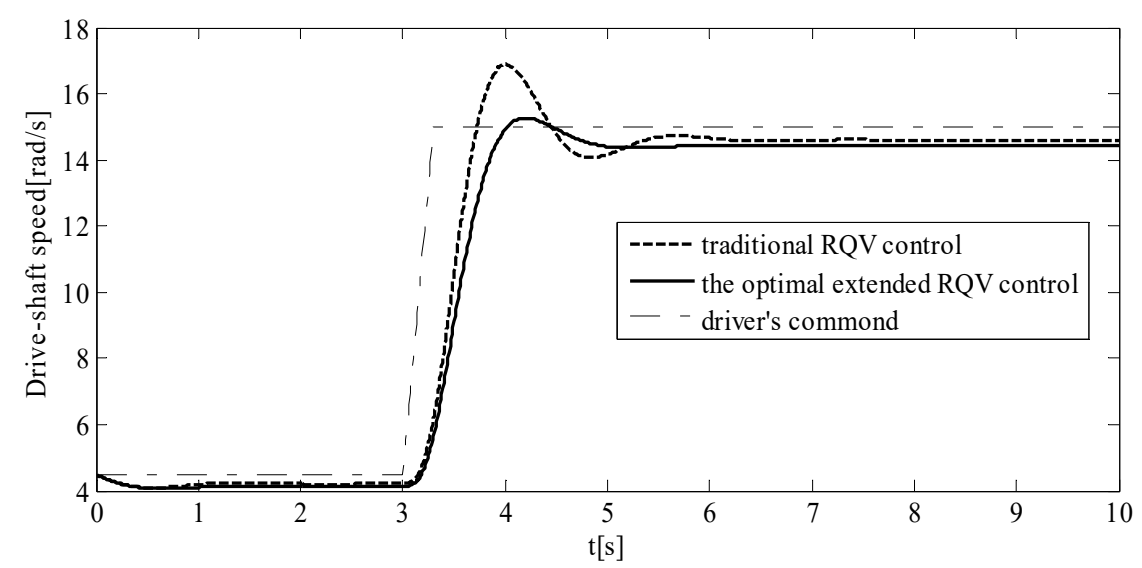

Figure 4 Motor rotor speed oscillation

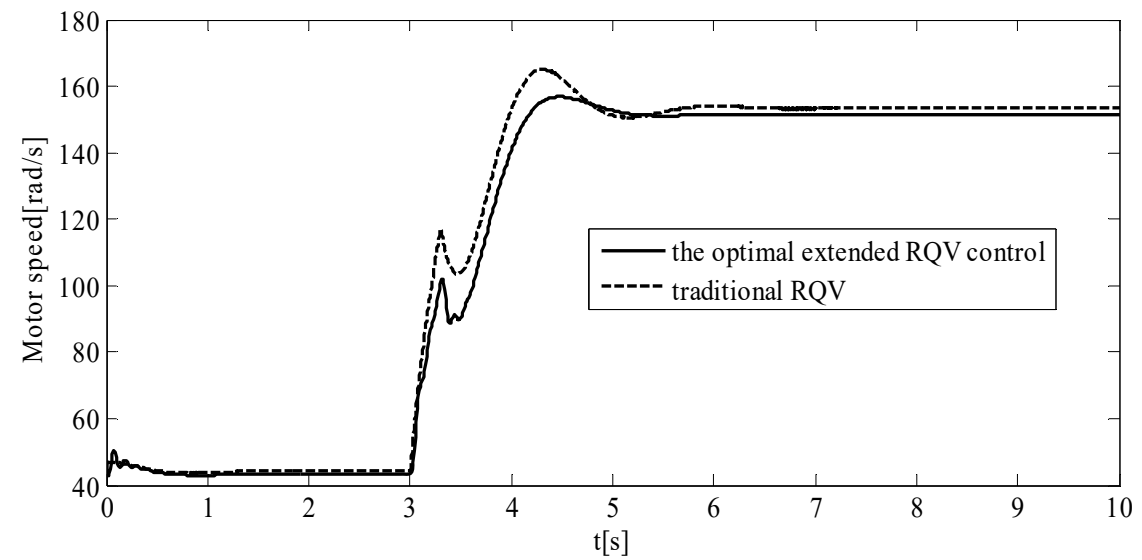

Figure 5 Torque in engaged gears of transmission

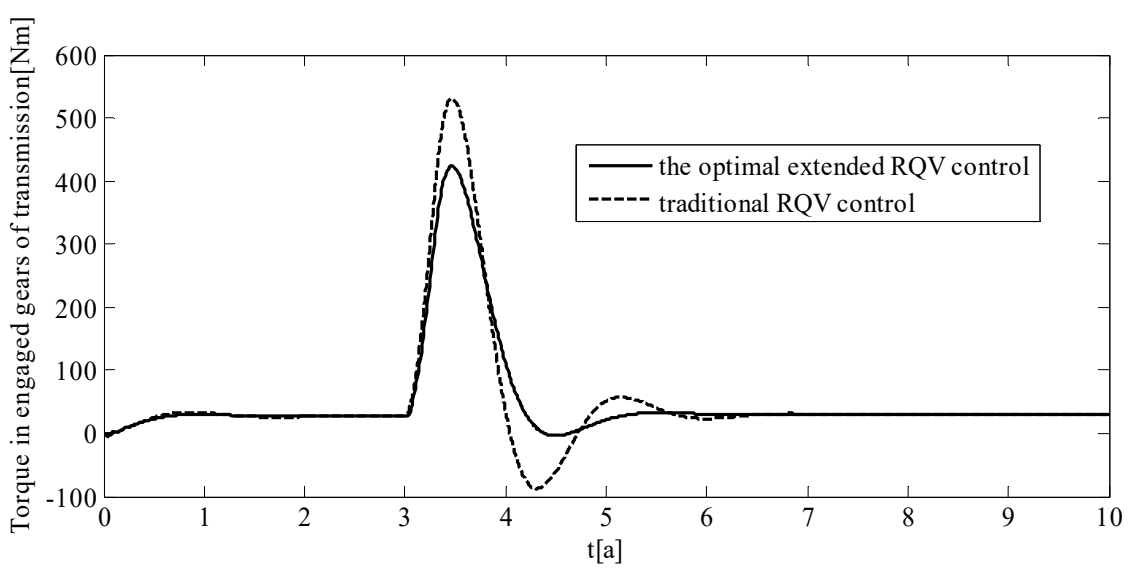


Figure 6 Motor output torque

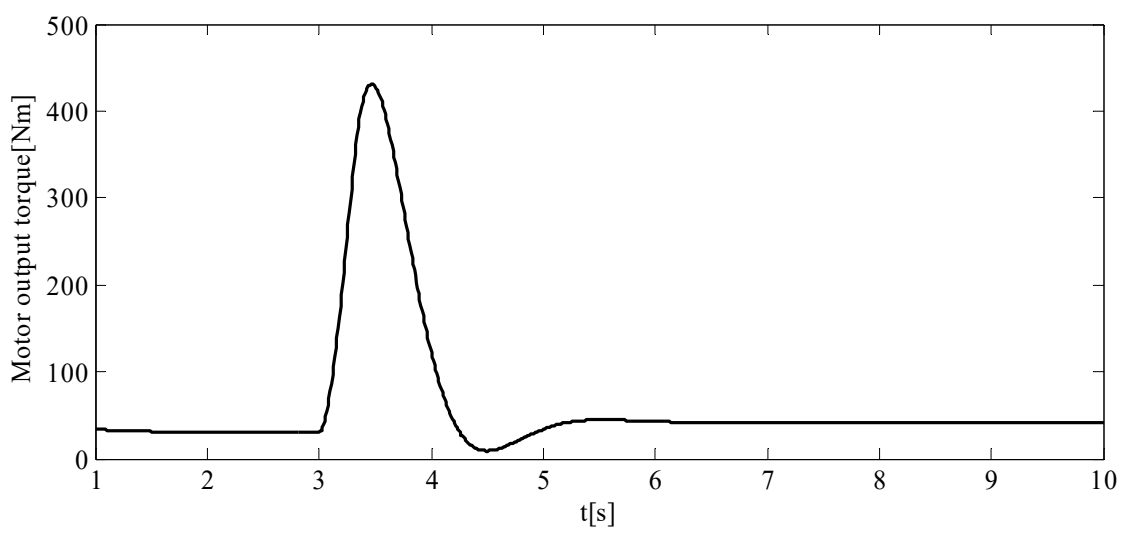

Figure 3 shows the driver's sudden command to change the speed at 3rd second, and a comparison between drive-shaft speed oscillation with the proposed optimal extended RQV control for motor output torque, and that of the conventional RQV. Figure 4 and Figure 5 are the comparisons of motor rotor speed oscillations and torque in engaged gears of transmission respectively with or without the optimal extended RQV control. Figure 6 is the control law of motor output torque. When a step change in acceleration is given by the driver, the EV powertrain system have a step change input, the powertrain system without control is a second-order oscillation system, oscillation of output variable $\theta$ is inevitable, and it will make the vehicle judders. It is obvious from these results that the powertrain with the proposed active damping oscillation controller has a better performance than that with the conventional RQV control in terms of less overshoot, less oscillation, and faster convergence. The obtained results indicate that the powertrain with the active damping system will provide passengers a comfort ride experience to a large extent, because passengers will be most sensitive to the jerking of vehicle body when shifting and accelerating. Furthermore, by active motor output torque control, torque in the engaged gears of transmission also be reduced significantly, and gradient of the peak value of variable torque is also reduced, so that there will be less shock on the teeth of transmission gear, which will further reduce the chances of fault and extend maintenance cycle.

\section{Conclusions}

In this paper a kinetic model of electric vehicle is established based on the Newton's second law. Because there is no clutch in the powertrain as an absorber and EV is equipped with a motor which can quickly respond to step change signal given by the driver, wheel speed oscillation will occur with no doubt. Hence, an active damping controller is proposed to mitigate the oscillations.

The principle of active damping is to control the torque output of motor opposing to speed oscillation trend in powertrain, the control law is calculated according to the powertrain motion state by optimal extended RQV controller, so that deviation from the expected speed is minimised. Simulation results and the comparisons show that the EV powertrain with the extended RQV controller has better dynamic properties with less 
oscillation, less overshoot and faster convergence in the motor rotor and drive-shaft, so that the ride comfort of passengers will be improved. The optimal extended RQV prevents the motor output torque to oscillate in a large scale, and gradient of the peak value of variable torque is reduced. Therefore, the torque oscillations and shock of engaged transmission gears will also be reduced, so comprehensive dynamic performance of $\mathrm{EV}$ powertrain can be improved.

\section{Acknowledgements}

This paper is supported by the National Natural Science Foundation of China (51575044).

\section{References}

Bang, J.S., Ko, Y.K. and Jung, T.H. (2015) The Active Damping Control to Reduce Driveline Oscillations for Electric Vehicles Using Wheel Speeds, SAE Technical Paper, doi:10.4271/2015-01-1113.

Baumann, J., Torkzadeh, D.D., Ramstein, A., Kiencke, U. and Schlegl, T. (2006) 'Model-based predictive anti-jerk control', Control Engineering Practice, Vol. 14, No. 3, pp.259-266.

Berriri, M., Chevrel, P. and Lefebvre, D. (2008) 'Active damping of automotive powertrain oscillations by a partial torque compensator', Control Engineering Practice, Vol. 16, No. 7, pp.874-883.

Caruntu, C.F., Lazar, M. and Cairano, S.D. (2016) 'Driveline oscillations damping: a tractable predictive control solution based on a piecewise affine model', Nonlinear Analysis: Hybrid Systems, Vol. 19, pp.168-185.

Fu, H., Tian, G.Y., Chen, H.X. and Chen, Q.S. (2010) 'A study on the torsional vibration control of motor-transmission integrated drive system', Automotive Engineering, Vol. 32, No. 7, pp.596-600.

Mitschke, M. and Wallentowitz, H. (2009) Tire, pp.6-10, translated by Chen, M.S. and Y.Q., Vehicle Dynamics, 4th ed., Tsinghua University Press, Springer, Beijing.

Mousavi, M.S.R., Pakniyat, A. and Boulet, B. (2014) 'Dynamic modeling and controller design for a seamless two-speed transmission for electric vehicles', IEEE International Conference on Control Applicants, pp.635-640.

Pettersson, M. (1997) Driveline Modeling and Control, Published PhD Dissertation, Linköping University, Linköping, Sweden.

Pham, T. and Bushnell, L. (2015) 'Two-degree-of-freedom damping control of driveline oscillations caused by pedal tip-in maneuver', 2015 American Control Conference, pp.1425-1432.

Song, Z.Y., Li, J.Q. and Shuai, Z.B. (2015)'Fuzzy logic torque control system in four-wheel-drive electric vehicles for active damping vibration control', International Journal of Vehicle Design, Vol. 68, Nos. 1-3, SI, pp.55-80.

Templin, P. and Egardt, B.(2011) 'A powertrain LQR-torque compensator with backlash handling', Oil \& Gas Science and Technology - Revue d'IFP Energies nouvelles, Vol. 66, No. 4, pp.645-654.

Yu, P., Zhang, T., Chen, S.Y. and Guo, R. (2015) Optimization of EV Mounting System Considering Power Train Torsion Control, SAE Technical Paper, doi:10.4271/2015-01-2225.

Zhang, H., Wang, C.L., Zhang, Y. and Yin, C.L. (2014) 'Active damping control for an electric vehicle using robust controller’, Applied Mechanics \& Materials, Vols. 475-476, pp.603-608. 